\title{
Ueber die asymptotischen Werthe einiger zahlentheoretischer
} Functionen.

Von

Edmund Landau in Berlin.

Es bezeichne $\mu(k)$ diejenige zahlentheoretische Function, welche

1) für $k=1$ gleich 1 ist,

2) wenn eine von 1 verschiedene Quadratzahl in $k$ aufgeht, gleich 0 ist,

3) wenn $k$ quadratfrei ist und aus $\omega$ Primfactoren besteht, gleich $(-1)^{\omega}$ ist.

Die Behauptung, dass die unendliche Reihe $\sum_{k=1}^{\infty} \frac{\mu(k)}{k}$ convergire und die Summe 0 habe, das heisst, dass die Function

$$
g(x)=\sum_{k=1}^{x} \frac{\mu(k)}{k}
$$

für $x=\infty$ den Grenzwerth 0 habe, ist ohne strengen Beweis schon von Euler*) ausgesprochen worden. Nachdem Herr Gram**) den Nachweis geführt hatte, dass die Function $g(x)$ jedenfalls nur zwischen zwei endlichen Unbestimmtheitsgrenzen oscilliren kann, hat Herr von Mangoldt***) die Convergenz der Reihe, also den Satz

$$
\lim _{x=\infty} g(x)=0
$$

bewiesen. Damit ergab sich auch das erste nicht triviale Resultat über eine andere Function

*) „Introductio in analysin infinitorum“‘ T. 1, Cap. 15, Nr. 277, Ex. 1, p. 229, Lausanne, 1748.

**) Unders $\varnothing$ gelser angaaende Maengden af Primtal under en given Graense", Det Kongelige Danske Videnskabernes Selskabs Skrifter, 6. Raekke, naturvidenskabelig og mathematisk Afdeling, II, 1884, S. 198 und 291.

\#Beweis der Gleichung $\sum_{k=1}^{\infty} \frac{\mu(k)}{k}=0 “$, Sitzungsberichte der Königlich Preussischen Akademie der Wissenschaften zu Berlin, 1897, S. 835-852. 


$$
f(x)=\sum_{k=1}^{x} \frac{\mu(k) \log k}{k}
$$

von der Möbius*) ohne strengen Beweis behauptet hatte, dass sie sich für $x=\infty$ der Grenze -1 nähere. $\mathrm{Da}$ nämlich zwischen "den Functionen $f(x)$ und $g(x)$ die von Herm von Mangoldt**) aufgestellte Beziehung besteht:

$$
|\log x g(x)-f(x)| \leqq 3+C,
$$

wo $C$ die Euler'sche Constante bezeichnet, so ergiebt sich aus (1)

anders geschrieben:

$$
\lim _{x=\infty} \frac{f(x)}{\log x}=0
$$

$$
f(x)=\{\log x\}
$$

wenn unter $\{F(x)\}$ eine Function verstanden wird, deren Quotient durch $F(x)$ für $x=\infty$ den Grenzwerth 0 hat. $O(F(x))$ bezeichne im Folgenden eine Function, deren Quotient durch $F(x)$ für grosse $x$ nicht beliebig grosser Werthe fähig ist, also zwischen zwei endlichen Unbestimmtheitsgrenzen oscillirt, mögen dieselben verschieden sein oder in 0 oder einem anderen Werthe zusammenfallen.

In meiner Doctordissertation****), welche, wie die erwähnte Arbeit Herrn von Mangoldts, sich auf die von den Herren Hadamard $\dagger$ ) und de la Vallée-Poussin †) gefundenen Ergebnisse aus der Theorie der Riemann'schen $\xi$-Function stützt, bin ich auf einem einfacheren Wege zu der Euler'schen Gleichung (1) gelangt, indem ich direct die asymptotische Gleichung (3) nachgewiesen habe, woraus (1) unter Anwendung der Ungleichheitsbedingung (2) folgt.

Die bedeutenden auf die Vertheilung der Nullstellen der Riemann'schen Function $\zeta(s)$ bezüglichen Resultate, zu denen Herr de la ValléePoussin $+f_{\dagger}$ ) in neuester Zeit gelangt ist, gestatteten diesem, den Nachweis* + )

*) "Ueber eine besondere Art von Umkehrung der Reihen", Journal für die reine und angewandte Mathematik, Bd. 9, 1832, S. 122.

$* *)$ 1. c., S. 839 .

**), Neuer Beweis der Gleichung $\sum_{k=1}^{\infty} \frac{\mu(k)}{k}=0^{*}$, Berlin, 1899.

†) „Sur la distribution des zéros de la fonction $\xi(s)$ et ses conséquences arithmétiques", Bulletin de la société mathématique de France, t. 24, 1896.

+1) "Recherches analytiques sur la théorie des nombres premiers", Annales de la société scientifique de Bruxelles, t. 20, $2^{\mathrm{e}}$ partie, 1896.

计) „Sur la fonction $\zeta(s)$ de Riemann et le nombre des nombres premiers inférieurs à une limite donnée", Mémoires couronnés et autres mémoires publiés par l'académie royale de Belgique, t. 59, 1899.

*) 1. c., 6 tes Kapitel, S. $63-67$. 
zu führen, dass $g(x)$ höchstens von der Grössenordnung $\frac{1}{\log x}$ ist, das heisst, dass eine endliche Zahl $h$ existirt, für welche identisch

$$
\log x|g(x)| \leqq h
$$

ist. Herr de la Vallée-Poussin hat also, wie sich aus einer Vergleichung der Relationen (2) und (4) ergiebt, den Nachweis geführt, dass $f(x)$ für alle $x$ zwischen zwei endlichen Grenzen enthalten ist,

$$
|f(x)| \leqq H \text {. }
$$

Darauf habe ich*) unter Anwendung der neuen Sätze Herrn de la Vallée-Poussin's über die Vertheilung der Primzahlen den Nachweis der Gleichung

$$
\lim _{x=\infty} f(x)=-1 \text {, }
$$

also den Convergenzbeweis der Reihe $\sum_{k=1}^{\infty} \frac{\mu(k) \log k}{k}$ erbracht; damit ist, wie sich aus (2) ergiebt, bewiesen, dass

$$
\lim _{x=\infty} \sup (\log x g(x)) \leqq 4+C
$$

ist, während in der (allerdings für sämmtliche $x$ giltigen) Ungleichheitsbedingung (4) die Constante $h$ einen sehr grossen Werth hat.

Da die beiden für $\mathfrak{R}(s)>1$ convergenten Reihen $\sum_{k=1}^{\infty} \frac{1}{k^{s}}$ and $\sum_{k=1}^{\infty} \frac{\mu(k)}{k^{s}}$ durch die Relation

$$
\sum_{k=1}^{\infty} \frac{1}{k^{s}} \cdot \sum_{k=1}^{\infty} \frac{\mu(k)}{k^{s}}=1
$$

verbunden sind und die Summe der ersten $x$ Glieder der ersteren für $s=1$ von der Ordnung $\log x$ ist, so könnte man vermuthen, dass die Summe $g(x)$ der ersten $x$ Glieder der Reihe $\sum_{k=1}^{\infty} \frac{\mu(k)}{k}$ von der Ordnung $\frac{1}{\log x}$ ist. Euler hatte - unerlaubterweise - die Folgerung

$$
\lim _{x=\infty} \sum_{k=1}^{x} \frac{\mu(k)}{k}=0
$$

daraus gezogen, dass das unendliche, auf alle Primzahlen erstreckte Product $\prod_{p}\left(1-\frac{1}{p}\right)$ wegen der Divergenz der Reihe $\sum_{p} \frac{1}{p}$ den Werth 0 hat, also daraus, dass

*) "Contribution à la théorie de la fonction $\zeta(s)$ de Riemann", Comptes rendus des séances de l'académie des sciences, Paris, t. 129, 1899, S. 812-815. 


$$
\lim _{x=\infty} \prod_{p \leq x}\left(1-\frac{1}{p}\right)=\lim _{x=\infty} \sum_{k} \frac{\mu(k)}{k}=0
$$

ist, wo $k$ alle Zahlen durchläuft, deren Primfactoren sämmtlich $\leqq x$ sind; der Euler'sche Satz selbst hat sich als richtig herausgestellt. Da nun Herr Mertens*) bewiesen hat, dass $\prod_{p \leqq x}\left(1-\frac{1}{p}\right)$ von der Ordnung $\frac{1}{\log x}$ ist, so könnte man erwarten, dass dies auch die Ordnung von $g(x)$ ist. Ich werde jedoch im ersten Paragraphen dieser Arbeit elementar nachweisen, dass also $\log x g(x)$ sich überhaupt keiner von 0 verschiedenen Grenze nähern kann, dass also $\log x g(x)$ sich entweder der Grenze 0 nähert oder - wenn $g(x)$ genau die Ordnung $\frac{1}{\log x}$ hat - zwischen zwei nicht zusammenfallenden und zufolge der Ungleichheitsbedingung (4) endlichen Unbestimmtheitsgrenzen oscillirt. Im $\$ 2$ werde ich unter Anwendung der Gleichung (6) zeigen, dass der erste dieser beiden denkbaren Fälle eintritt, dass also

$$
\lim _{x=\infty}(\log x g(x))=\lim _{x=\infty}\left(\log x \sum_{k=1}^{x} \frac{\mu(k)}{k}\right)=0
$$

ist. Im $\S 3$ werden genauere Abschätzungen der beiden Functionen $f(x)$ und $g(x)$ gegeben und im $\S 4$ einige Folgerungen aus den gefundenen Sätzen gezogen werden.

\section{$\S 1$.}

Würde sich $\log x g(x)$ für $x=\infty$ einer positiven Grenze $2 c$ nähern, so wäre für alle $x$ oberhalb einer gewissen Zahl $n$

es wäre also für $\nu \leqq \frac{x}{n}$

$$
g(x) \geq \frac{c}{\log x}
$$

also

$$
g\left(\frac{x}{v}\right) \geqq \frac{e}{\log x-\log v}
$$

$$
\sum_{v=1}^{\left[\frac{x}{n}\right]} \frac{1}{\nu} g\left(\frac{x}{v}\right) \geqq \sum_{v=1}^{\left[\frac{x}{n}\right]} \frac{1}{v} \frac{e}{\log x-\log v}
$$

ein Ausdruck, der mit einem für $x=\infty$ zwischen endlichen Grenzen befindlichen Fehler durch das Integral

* „Ein Beitrag zur analytischen Zahlentheorie", Journal für die reine und angewandte Mathematik, Bd. 78, 1874, S. 53. 


$$
\begin{aligned}
c \int_{1}^{\frac{2}{n}} \frac{1}{v} \frac{d v}{\log x-\log v}=c \int_{1}^{\frac{x}{n}} \frac{d \log v}{\log x-\log v} & =c[-\log (\log x-\log v)]_{1}^{\frac{x}{n}} \\
& =c(\log \log x-\log \log x)
\end{aligned}
$$

ersetzt werden kann, also jede Grenze überschreitet. Dies steht jedoch mit der von Herrn Gram*) angegebenen Identität

in Widerspruch; denn wegen

$$
1=\sum_{\nu=1}^{x} \frac{1}{\nu} g\left(\frac{x}{\nu}\right)=\sum_{1}^{\left[\frac{x}{n}\right]} \frac{1}{\nu} g\left(\frac{x}{\nu}\right)+\sum_{\left[\frac{x}{n}\right]+1}^{x} \frac{1}{v} g\left(\frac{x}{\nu}\right)
$$

ist

$$
|g(y)| \leqq 1 *)
$$

$$
\left|\sum_{\left[\frac{x}{n}\right]+1}^{x} \frac{1}{v} g\left(\frac{x}{v}\right)\right| \leqq \sum_{\left[\frac{x}{n}\right]+1}^{x} \frac{1}{v} \leqq \log x+1-\log \left[\frac{x}{n}\right]<\log n+2,
$$

also für alle $x$ zwischen zwei endlichen Grenzen enthalten, so dass thatsächlich auch $\sum_{1}^{\left[\frac{x}{n}\right]} \frac{1}{v} g\left(\frac{x}{v}\right)$ endlich bleiben muss.

Die Annahme, dass $\log x g(x)$ sich einer negativen Grenze nähert, führt ebenso zu einem Widerspruch.

$\log x g(x)$ nähert sich also entweder der Grenze 0 oder keiner Grenze.

\section{$\S 2$.}

Der Ausgangspunkt des im Folgenden zu erbringenden Nachweises der Behauptung

$$
\lim (\log x g(x))=0
$$

ist die von Herrn Cesàro***;) aufgestellte Identität

$$
\sum_{t=1}^{x} \frac{1}{t} \sum_{\nu} \mu\left(\frac{t}{\nu}\right) \log \nu=\sum_{p, m} \frac{\log p}{p^{m}}
$$

wo links $\nu$ alle Theiler von $t$ durchläuft, rechts $p$ alle Primzahlen und $m$

*) l. c., S. 197, Gleichung (43), wo $r=1$ zu setzen ist.

*) S. Gram, 1. c., S. 198 und 291, v. Mangoldt, 1. c., S. 83\%.

***) „Sur diverses questions d'arithmétique“, Mémoires de la société royale des sciences de Liège, $2^{e}$ série, t. 10, 1883; es ist die Gleichung (12), S. 318 mit der Bemerkung auf S. 320, Z. 7-6 v. u. zu combiniren. 
alle positiven ganzen Zahlen, für welche $p^{m} \leqq x$ ist. Die Quelle dieser Identität ist der Umstand, dass die Summe $\sum_{\nu} \mu\left(\frac{t}{\nu}\right) \log \nu$ verschwindet, wenn $t$ keine Primzahlpotenz ist, dagegen gleich $\log p$ ist, wenn $t$ einePrimzahlpotenz $p^{m}$ ist. Wie man aus der Zerlegung $t=\frac{t}{v} \cdot v$ leicht sieht, ist die linke Seite von (8) mit der Summe*)

$$
\sum_{\nu=2}^{x} \frac{\log v}{\nu} g\left(\frac{x}{\nu}\right)=\sum_{\nu=2}^{x} \frac{\log v}{\nu} \sum_{k=1}^{\frac{x}{v}} \frac{\mu(k)}{k}
$$

identisch; denn $\nu$ und $\frac{t}{\nu}=k$ durchlaufen unabhängig alle Zahlen, für welche $\nu k \leqq x$ ist.

Im Folgenden werde ich eine dritte Form derselben benutzen, nämlich

$$
\sum_{k=1}^{\frac{x}{2}} \frac{\mu(k)}{k} \sum_{\nu=2}^{\frac{x}{k}} \frac{\log \nu}{\nu}
$$

und diese Summe werde ich in ähnlicher Weise behandeln wie Herr von Mangoldt**) die analoge Summe $\sum_{k=1}^{x} \frac{\mu(k)}{k} \sum_{\nu=1}^{\frac{x}{k}} \frac{1}{v}$, deren Betrachtung ihn zu der Ungleichheitsbedingung (2) geführt hat.

Es ist für ganzzahlige Werthe von $y$

$$
\sum_{\nu=2}^{y} \frac{\log v}{v}=\int_{1}^{y} \frac{\log v}{v} d v+D+O\left(\frac{\log y}{y}\right)=\frac{1}{2} \log ^{2} y+D+O\left(\frac{\log y}{y}\right),
$$

wo $D$ eine Constante bezeichnet, auf deren Werth es im Folgenden nicht ankommt. Dies gilt auch für gebrochene $y$, da

$$
\int_{[y]}^{y} \frac{\log v}{\nu} d \nu=O\left(\frac{\log y}{y}\right)
$$

ist. Daher ergiebt sich

*) Die Summationsgrenzen brauchen im Folgenden durchweg keine ganzen Zahlen zu sein; der Summationsbuchstabe hat alle im Summationsintervall mit Einschluss der Grenzen enthaltenen ganzzahligen Werthe zu durchlanfen.

**) 1. c. S. 838 . 
Da

$$
\sum_{k=1}^{\frac{x}{2}} \frac{\mu(k)}{k} \sum_{\nu=2}^{\frac{x}{k}} \frac{\log v}{\nu}=\sum_{k=1}^{\frac{x}{2}} \frac{\mu(k)}{k}\left(\frac{1}{2} \log ^{2} \frac{x}{k}+D+O\left(\frac{k}{x} \log \frac{x}{k}\right)\right) .
$$

ferner

$$
\begin{aligned}
\left|\sum_{k=1}^{\frac{x}{2}} \frac{\mu(k)}{k} O\left(\frac{k}{x} \log \frac{x}{k}\right)\right| & =\frac{1}{x} O \sum_{k=1}^{x} \log \frac{x}{k}=\frac{1}{x} O([x] \log x-\log ([x] !)) \\
& =\frac{1}{x} O(x \log x+O(\log x)-x \log x+x)=O(1),
\end{aligned}
$$

und

$$
\sum_{k=1}^{\frac{x}{2}} \frac{\mu(k)}{k} D=D g\left(\frac{x}{2}\right)=O(1)
$$

$$
\sum_{k=\left[\frac{x}{2}\right]+1}^{x} \frac{\mu(k)}{k} \frac{1}{2} \log ^{2} \frac{x}{k}=O \sum_{k=\left[\frac{x}{2}\right]+1}^{x} \frac{1}{k}=O(1)
$$

ist, so ist die Summe (9), also die linke Seite der Gleichung (8) mit einem für $x=\infty$ endlich bleibenden Fehler durch

$$
\begin{aligned}
\frac{1}{2} \sum_{k=1}^{x} \frac{\mu(k)}{k} \log ^{2} \frac{x}{k} & =\frac{1}{2} \log ^{2} x \sum_{k=1}^{x} \frac{\mu(k)}{k}-\log x \sum_{k=1}^{x} \frac{\mu(k)}{k} \log k \\
& +\frac{1}{2} \sum_{k=1}^{x} \frac{\mu(k)}{k} \log ^{2} k
\end{aligned}
$$

ersetzbar.

Andererseits hat Herr Mertens*) nachgewiesen, dass die rechte Seite der Gleichung (8) mit einem für $x=\infty$ endlich bleibenden Fehler gleich $\log x$ ist. Es ergiebt sich also

$$
\frac{1}{2} \log ^{2} x g(x)-\log x f(x)+\frac{1}{2} \sum_{k=1}^{x} \frac{\mu(k) \log ^{2} k}{k}=\log x+O(1)
$$

Es ist zu beachten, dass zur Aufstellung dieser Relation nur elementare, d. h. von der Theorie der Functionen complexen Argumentes und insbesondere von der Theorie der $\xi$-Function unabhängige Hilfsmittel herangezogen worden sind; nunmehr mache ich jedoch von dem Satze Gebrauch, dass

$$
\lim _{x=\infty} f(x)=-1
$$

ist. Ich beweise zunächst den

*) 1. c., S. 49. 


\section{Hilfssatz:}

$$
\sum_{k=1}^{x} \frac{\mu(k) \log ^{2} k}{k}=\{\log x\}
$$

Beweis: Es ist

$$
\begin{aligned}
\sum_{k=1}^{x} \frac{\mu(k) \log ^{2} k}{k} & =\sum_{k=1}^{x} \frac{\mu(k) \log k}{k} \log k=\sum_{k=1}^{x}(f(k)-f(k-1)) \log k \\
& =\sum_{k=1}^{x} f(k)(\log k-\log (k+1))+f(x) \log ([x]+1) \\
& =-\sum_{k=1}^{x} f(k) \log \left(1+\frac{1}{k}\right)+f(x) \log x+O\left(\frac{1}{x}\right) \\
& =-\sum_{k=1}^{x} f(k)\left(\frac{1}{k}-\frac{\vartheta_{k}}{2 k^{2}}\right)+f(x) \log x+O\left(\frac{1}{x}\right)\left(0 \leqq \vartheta_{k} \leqq 1\right)
\end{aligned}
$$

Offenbar convergirt $\sum_{k=1}^{\infty} f(k) \frac{\vartheta_{k}}{2 k^{2}} ;$ ferner ist

$$
f(x) \log x=-\log x+\{1\} \cdot \log x=-\log x+\{\log x\}
$$

also

$$
\sum_{k=1}^{x} \frac{\mu(k) \log ^{2} k}{k}=-\sum_{k=1}^{x} \frac{f(k)}{k}-\log x+\{\log x\}
$$

$\delta$ sei eine beliebig kleine positive Grösse. Dann ist $n$ so bestimmbar, dass für alle $k \geqq n$

ist; also ist

$$
|1+f(k)| \leqq \frac{\delta}{2}
$$

$$
\left|\sum_{k=1}^{x} \frac{1+f(k)}{k}\right| \leqq\left|\sum_{k=1}^{n-1} \frac{1+f(k)}{k}\right|+\left|\sum_{k=n}^{x} \frac{1+f(k)}{k}\right| \leqq a+\frac{\delta}{2} \sum_{k=n}^{x} \frac{1}{k},
$$

wo $a$ eine endliche nur von $\delta$ abhängige Zahl bezeichnet, die also für hinreichend grosse $x$ kleiner als $\frac{\delta}{2} \log x$ ist. Da für $n \geqq 2$

$$
\sum_{k=n}^{x} \frac{1}{k} \leqq \log x
$$

ist, ist also für hinreichend grosse $x$ 


$$
\begin{aligned}
\left|\sum_{k=1}^{x} \frac{1+f(k)}{k}\right| & \leqq \frac{\delta}{2} \log x+\frac{\delta}{2} \log x=\delta \log x, \\
\sum_{k=1}^{x}\left(\frac{1}{k}+\frac{f(k)}{k}\right) & =\{\log x\} \\
\sum_{k=1}^{x} \frac{f(k)}{k}=-\sum_{k=1}^{x} \frac{1}{k}+\{\log x\}=-\log x+\{\log x\} & \\
\sum_{k=1}^{x} \frac{\mu(k) \log ^{2} t}{k} & =\log x+\{\log x\}-\log x+\{\log x\}=\{\log x\},
\end{aligned}
$$

was zu beweisen war.

Die Gleichung (10) ergiebt also

das heisst

$$
\begin{aligned}
\frac{1}{2} \log ^{2} x g(x)+\log x+\{\log x\} & =\log x+O(1), \\
\log ^{2} x g(x) & =\{\log x\}, \\
\log x g(x) & =\{1\},
\end{aligned}
$$

$$
\lim _{x=\infty}\left(\log x \sum_{k=1}^{x} \frac{\mu(k)}{k}\right)=0 .
$$

Man kann auch auf folgendem Wege, gleichfalls unter Benutzung des soeben bewiesenen Hilfssatzes (11), zu dieser Gleichung gelangen, indem man ihn statt mit (10) mit der von Herrn von Mangoldt*) aufgestellten Gleichung

$$
\log ^{2} x g(x)-\sum_{k=1}^{x} \frac{\mu(k) \log ^{2} k}{k}=\{\log x\}
$$

combinirt. Aus (11) und (12) folgt nämlich durch Elimination von $\sum_{k=1}^{x} \frac{\mu(k) \log ^{2} k}{k}$

$$
\begin{gathered}
\log ^{2} x g(x)-\{\log x\}=\{\log x\}, \\
\log x g(x)=\{1\} .
\end{gathered}
$$

Der zuerst gegebene Beweis verdient jedoch den Vorzug, weil die Gleichung (12) bisher im Gegensatz zu (10) nicht elementar bewiesen werden kann. Thre Herleitung ist der einzige Theil des v. Mangoldt'schen Con-

7) 1. c., S. 845 . 
vergenzbeweises der Reihe $\sum_{k=1}^{\infty} \frac{\mu(k)}{k}$, in welchem von der Theorie der ६-Function Gebrauch gemacht wird.

Eine dritte Beweisanordnung ergiebt sich durch Combination von (10) und (12). Die Elimination von $\sum_{k=1}^{x} \frac{\mu(k) \log ^{2} k}{k}$ ergiebt hier

$$
\begin{gathered}
\log ^{2} x g(x)-\log x f(x)=\log x+\{\log x\}, \\
\log x g(x)-f(x)=1+\{1\} .
\end{gathered}
$$

Die Gleichung (13) sagt aus, dass die Function

$$
\log x \sum_{k=1}^{x} \frac{\mu(k)}{k}-\sum_{k=1}^{x} \frac{\mu(k) \log k}{k}
$$

von der Herr v. Mangoldt*) zuerst bewiesen hat, dass sie zwischen endlichen Grenzen enthalten bleibt, sich für $x=\infty$ der Grenze 1 nähert, und es ist bemerkenswerth, dass sich dies ohne Zuhilfenahme anderer transcendenter Hilfsmittel ergeben hat als der in der v. Mangoldt'schen Arbeit enthaltenen.

Aus (13) folgt unter Benutzung von'

der behauptete Satz

$$
f(x)=-1+\{1\}
$$

$$
\log x g(x)=\{1\}
$$

\section{$\S 3$.}

Am erwähnten Orte $\left.{ }^{* *}\right)$ habe ich mich damit begnügt, den Nachweis der Convergenz der Reihe $\sum_{k=1}^{\infty} \frac{\mu(k) \log k}{k}$ zu führen. Die dort angestellten Betrachtungen will ich nunmehr in eine solche Form bringen, dass sie zugleich eine Abschätzung des Restes - $(1+f(x))$ der Reihe liefern, der durch Weglassung der ersten $x$ Glieder entsteht. Ersetzt man in $\sum_{k=1}^{x} \frac{\mu(k) \log k}{k}$ für $k=\prod_{p} p^{\alpha}$ den Factor $\log k$ durch $\sum_{p} \alpha \log p$ und berücksichtigt, dass wegen des Factors $\mu\left(k_{i}\right)$ nur solche $k$ vorkommen,

*) 1. c., S. 839.

*) "Contribution etc.“ 
in denen jeder Primfactor genau einmal aufgeht, so ist, da $k$ alle Vielfachen von $p$ durchläuft,

woraus sich leicht

$$
\left.f(x)=\sum_{k=1}^{x} \frac{\mu(k) \log k}{k}=\sum_{p \leq x} \log p \sum_{m=1}^{\frac{x}{p}} \frac{\mu(m p)}{m p} *\right),
$$

ergiebt.

$$
\begin{aligned}
-f(x) & =\sum_{p \leq x} \log p\left(\frac{1}{p} \sum_{1}^{p} \frac{\mu(k)}{k}+\frac{1}{p^{2}} \sum_{1}^{\frac{x}{p^{2}}} \frac{\mu(k)}{k}+\frac{1}{p^{3}} \sum_{1}^{\frac{x}{p^{3}}} \frac{\mu(k)}{k}+\cdots\right) \\
& =\sum_{p \leq x} \frac{\log p}{p} g\left(\frac{x}{p}\right)+\sum_{p \leq \sqrt{x}} \log p\left(\frac{1}{p^{2}} g\left(\frac{x}{p^{2}}\right)+\frac{1}{p^{3}} g\left(\frac{x}{p^{3}}\right)+\cdots\right)
\end{aligned}
$$

In die erste Summe führe ich die durch die Gleichungen

$$
\begin{gathered}
\varepsilon(0)=0, \\
\varepsilon(x)=\frac{\sum_{p \leq x} \log p}{x}-1=\frac{\vartheta(x)}{x}-1 \quad(x>0)
\end{gathered}
$$

erklärte Function $\varepsilon(x)$ ein. Von derselben hat Herr Mertens $*$ ) bewiesen, dass für alle $x$

$$
|\varepsilon(x)| \leqq 1
$$

ist und Herr de la Vallée-Poussin ***), dass

$$
\varepsilon(x)=O\left(e^{-b \sqrt{\log x}}\right)
$$

ist, wo $b$ eine positive Constante bezeichnet. Die Einführung der Function $\varepsilon(x)$ geschieht mit Hilfe der Identität

$$
\begin{aligned}
\vartheta(\nu)-\vartheta(\nu-1) & =\nu+\nu \varepsilon(\nu)-(\nu-1)-(\nu-1) \varepsilon(\nu-1) \\
& =1+\nu \varepsilon(\nu)-(\nu-1) \varepsilon(\nu-1)=\log \nu \text { oder } 0
\end{aligned}
$$

je nachdem $\nu$ prim oder zusammengesetzt ist; es ergiebt sich

$$
\begin{aligned}
\sum_{p \leq x} \frac{\log p}{p} g\left(\frac{x}{p}\right) & =\sum_{\nu=1}^{x} \frac{1+v \varepsilon(\nu)-(\nu-1) \varepsilon(\nu-1)}{\nu} g\left(\frac{x}{\nu}\right) \\
& =\sum_{\nu=1}^{x} \frac{1}{\nu} g\left(\frac{x}{\nu}\right)+\sum_{\nu=1}^{x}(\varepsilon(\nu)-\varepsilon(\nu-1)) g\left(\frac{x}{\nu}\right) \\
& +\sum_{v=1}^{x} \frac{\varepsilon(\nu-1)}{\nu} g\left(\frac{x}{\nu}\right)
\end{aligned}
$$

*) Die Anwendung des Summationsbuchstaben $p$ bezeichne, dass nur die Primzahlen des betreffenden Intervalles durchlaufen werden sollen.

*) 1. c., S. 48 .

*) „Sur la fonetion etc.", S. 54. 
also unter Anwendung der Gram'schen Identität

$$
\sum_{\nu=1}^{x} \frac{1}{\nu} g\left(\frac{x}{\nu}\right)=1
$$

und durch partielle Summation der zweiten Summe auf der rechten Seite von (15)

$$
\begin{aligned}
& \sum_{p \leq x} \frac{\log p}{p} g\left(\frac{x}{p}\right)=1+\sum_{\nu=1}^{x} \varepsilon(\nu)\left(g\left(\frac{x}{\nu}\right)-g\left(\frac{x}{\nu+1}\right)\right)+\sum_{\nu=1}^{x} \frac{\varepsilon(\nu-1)}{\nu} g\left(\frac{x}{\nu}\right), \\
& (16)-f(x)=1+\sum_{\nu=1}^{x} \varepsilon(\nu)\left(g\left(\frac{x}{\nu}\right)-g\left(\frac{x}{\nu+1}\right)\right)+\sum_{\nu=1}^{x} \frac{\varepsilon(\nu-1)}{\nu} g\left(\frac{x}{\nu}\right) \\
& +\sum_{p \leq \sqrt{x}} \log p\left(\frac{1}{p^{2}} g\left(\frac{x}{p^{2}}\right)+\frac{1}{p^{3}} g\left(\frac{x}{p^{3}}\right)+\cdots+\frac{1}{p^{\left[\frac{\log x}{\log p}\right]} g} g\left(\frac{x}{p^{\left[\frac{\log x}{\log p}\right]}}\right)\right) .
\end{aligned}
$$

1) Für die erste Summe der rechten Seite ergiebt sich unter Anwendung des von Herrn de la Vallée-Poussin*) bewiesenen Satzes

$$
g(x)=O\left(\frac{1}{\log x}\right)
$$

durch passende Zerlegung in drei Theile:

$1 \alpha)$

$$
\begin{aligned}
& \left|\sum_{1}^{\sqrt{\log x}} \varepsilon(\nu)\left(g\left(\frac{x}{\nu}\right)-g\left(\frac{x}{\nu+1}\right)\right)\right| \leqq \sum_{1}^{\sqrt{\log x}}\left(\left|g\left(\frac{x}{\nu}\right)\right|+\left|g\left(\frac{x}{\nu+1}\right)\right|\right) \\
& =O \sum_{1}^{\sqrt{\log x}}\left(\frac{1}{\log x-\log \nu}+\frac{1}{\log x-\log (\nu+1)}\right) \\
& =O \sum_{1}^{\sqrt{\log x}} \frac{1}{\log x-\log (\sqrt{\log x}+1)}=O\left(\frac{\sqrt{\log x}}{\log x}\right)=O\left(\frac{1}{\sqrt{\log x}}\right) .
\end{aligned}
$$

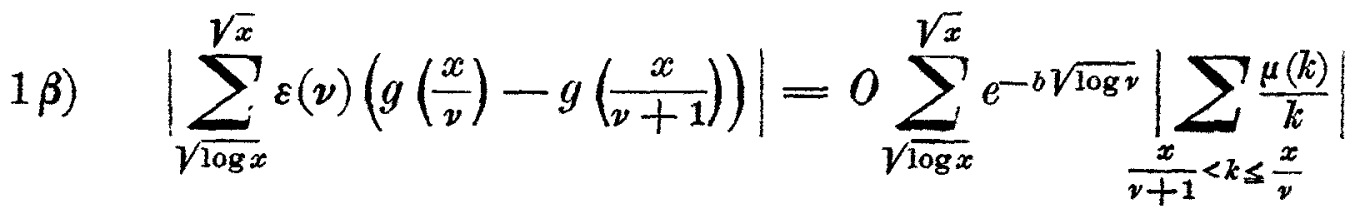

$$
\begin{aligned}
& =O \sum_{\sqrt{\log x}}^{\sqrt{x}} e^{-b \sqrt{\log v}} \sum_{\frac{x}{v+1}<k \leq \frac{x}{v}} \frac{1}{k}
\end{aligned}
$$

) „Sur la fonction etc.“, S. 67. 
Wegen $\nu^{2} \leqq x, \frac{v}{x} \leqq \frac{1}{\nu}$ ist

$$
\begin{aligned}
& \sum_{\frac{x}{\nu+1}<k \leq \frac{x}{v}} \frac{1}{k}=\sum_{1}^{\frac{x}{v}} \frac{1}{k}-\sum_{1}^{\frac{x}{v+1}} \frac{1}{k}=\log \frac{x}{\nu}+C+O\left(\frac{v}{x}\right)-\log \frac{x}{\nu+1}-C+O\left(\frac{\nu+1}{x}\right) \\
& =\log \left(1+\frac{1}{v}\right)+O\left(\frac{v}{x}\right) \leqq \frac{1}{v}+O\left(\frac{v}{x}\right)=O\left(\frac{1}{\nu}\right), \\
& \left|\sum_{\sqrt{\log x}}^{\sqrt{x}} \varepsilon(\nu)\left(g\left(\frac{x}{\nu}\right)-g\left(\frac{x}{\nu+1}\right)\right)\right|=O \sum_{\sqrt{\log x}}^{\sqrt{x}} \frac{e^{-b \sqrt{\log v}}}{\nu} \\
& =0 \int_{\sqrt{\log x}}^{\sqrt{x}} \frac{e^{-b \sqrt{\log \nu}}}{\nu} d \nu+O\left(\frac{e^{-b \sqrt{\log \sqrt{\log x}}}}{\sqrt{\log x}}\right) .
\end{aligned}
$$

Es ist

$$
\begin{aligned}
& \int_{\sqrt{\log x}}^{\sqrt{x}} \frac{e^{-b \sqrt{\log v}}}{\nu} d \nu<\int_{\sqrt{\log x}}^{\infty} \frac{e^{-b \sqrt{\log \nu}}}{\nu} d \nu=\int_{\frac{1}{2} \log \log x}^{\infty} e^{-b \sqrt{u}} d u \\
& =2 e^{-\frac{b}{\sqrt{2}} \sqrt{\log \log x}}\left(\frac{\sqrt{\log \log x}}{b \sqrt{2}}+\frac{1}{b^{2}}\right)=O\left(\sqrt{\log \log x} e^{-\frac{b}{\sqrt{2}} \sqrt{\log \log x}}\right) \\
& =O\left(e^{-c \sqrt{\log \log x}}\right),
\end{aligned}
$$

wo $c$ eine positive Constante bezeichnet.

$$
\begin{aligned}
& \text { 1y) }\left|\sum_{\sqrt{x}}^{x} \varepsilon(\nu)\left(g\left(\frac{x}{\nu}\right)-g\left(\frac{x}{\nu+1}\right)\right)\right|=0 \sum_{\sqrt{x}}^{x} e^{-b \sqrt{\log \nu}} \sum_{\frac{x}{\nu+1}<k \leq \frac{x}{\nu}} \frac{1}{k} \\
& =0 \sum_{\sqrt{x}}^{x} e^{-b \sqrt{\log \sqrt{x}}} \sum_{\frac{x}{\nu+1}<k \leq \frac{x}{\nu}} \frac{1}{k} \\
& \left.=e^{-\frac{b}{\sqrt{2}} \sqrt{\log x}} O \sum_{\nu=1}^{x} \sum_{\frac{x}{\nu+1}<k \leq \frac{x}{\nu}} \frac{1}{k}=e^{-\frac{b}{\sqrt{2}} \sqrt{\log x}} O \sum_{1}^{x} \frac{1}{k} *\right)=O\left(\log x e^{-\frac{b}{\sqrt{2}} \sqrt{\log x}}\right) .
\end{aligned}
$$

Wenn man das Glied höchster Ordnung in den unter $1 \alpha), 1 \beta), 1 \gamma$ ) gefundenen Theilresultaten beibehält, ist also

$$
\sum_{1}^{x} \varepsilon(\nu)\left(g\left(\frac{x}{\nu}\right)-g\left(\frac{x}{\nu+1}\right)\right)=O\left(e^{-c \sqrt{\log \log x}}\right) .
$$

*) Denn die Intervalle $\frac{x}{2} \cdots x, \frac{x}{3} \cdots \frac{x}{2}, \cdots$ ergeben zusammen $1 \cdots x$. 
2) Was die zweite Summe in (16) anbetrifft, so ist

$2 \alpha)$

$$
\begin{aligned}
\left|\sum_{1}^{\sqrt{\log x}} \frac{\varepsilon(v-1)}{v} g\left(\frac{x}{v}\right)\right| & =0 \sum_{1}^{\sqrt{\log x}} \frac{1}{v} \frac{1}{\log x-\log v} . \\
& =0 \sum_{1}^{\sqrt{\log x}} \frac{1}{v} \frac{1}{\log x-\log \sqrt{\log x}}=\frac{1}{\log x} O \sum_{1}^{\sqrt{\log x}} \frac{1}{v} \\
& =\frac{1}{\log x} O(\log \sqrt{\log x})=O\left(\frac{\log \log x}{\log x}\right) .
\end{aligned}
$$

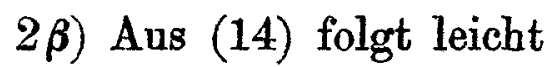

also

$$
|\varepsilon(\nu-1)|=O\left(e^{-b \sqrt{\log x}}\right),
$$

$$
\begin{aligned}
& \left|\sum_{\sqrt{\log x}}^{x} \frac{\varepsilon(\nu-1)}{\nu} g\left(\frac{x}{\nu}\right)\right|=0 \sum_{\sqrt{\log x}}^{x} \frac{e^{-x \sqrt{\log y}}}{\nu} \\
& =0 \int_{\sqrt{\log x}}^{e^{-b \sqrt{\log \nu}}} d \nu+O\left(\frac{e^{-b \sqrt{\log \sqrt{\log x}}}}{\nu}\right)=O\left(e^{-c \sqrt{\log \log x}}\right),
\end{aligned}
$$

wie in $1 \beta$ ).

3) Die dritte Summe in (16) ist dem absoluten Werthe nach

$$
\leqq \sum_{n} \frac{\log p}{n}\left|g\left(\frac{x}{n}\right)\right|
$$

wo $n$ alle Primzahlpotenzen*) bis $x$ oder auch bis $\infty$ durchläuft und $p$ die zugehörige Basis bezeichnet; es ergiebt sich durch passende Zerlegung in zwei Theile

$3 \alpha)$

$$
\begin{aligned}
& \sum_{n \leq \log x} \frac{\log p}{n}\left|g\left(\frac{x}{n}\right)\right|=0 \sum_{n \leq \log x} \frac{\log p}{n} \frac{1}{\log x-\log n} \\
= & O \sum_{n \leq \log x} \frac{\log p}{n} \frac{1}{\log x-\log \log x}=\frac{1}{\log x} O \sum_{n \leq \log x} \frac{\log p}{n} .
\end{aligned}
$$

Die auf alle Primzahlpotenzen erstreckte Reihe

$$
\sum_{n} \frac{\log p}{n}=\sum_{p} \log p\left(\frac{1}{p^{2}}+\frac{1}{p^{3}}+\cdots\right)=\sum_{p} \frac{\log p}{p(p-1)}
$$

convergirt; demnach ist

$$
\sum_{n \leq \log x} \frac{\log p}{p}\left|g\left(\frac{x}{n}\right)\right|=\frac{1}{\log x} O(1)=O\left(\frac{1}{\log x}\right) .
$$

*) mit Exponenten $\geqq 2$. 
$\mathbf{3} \beta)$

$$
\sum_{\log x \leq n} \frac{\log p}{p}\left|g\left(\frac{x}{n}\right)\right|=O \sum_{\log x \leq n} \frac{\log p}{n}=O\left(\frac{1}{\sqrt{\log x}}\right)
$$

da der Rest der convergenten Reihe $\sum_{n} \frac{\log p}{n}$

$$
\sum_{y \leq n} \frac{\log p}{n}=O\left(\frac{1}{\sqrt{y}}\right)
$$

ist, wie man leicht zeigen kann.

Alles in Allem erhalte ich also das Resultat

$$
f(x)=-1+O\left(e^{-c \sqrt{\log \log x}}\right) .
$$

Daraus ergiebt sich mit Hilfe der beim Beweise des Hilfssatzes auf S. 577 angestellten Betrachtungen

$$
\begin{aligned}
& \sum_{k=1}^{x} \frac{\mu(k) \log ^{2} k}{k}=-\sum_{k=1}^{x} \frac{f(k)}{k}+O(1)+f(x) \log x+O\left(\frac{1}{x}\right) \\
& \left.=-\sum_{k=3}^{x} \frac{-1+O\left(e^{-c \sqrt{\log \log k}}\right)}{k}-\log x+O\left(\log x e^{-c \sqrt{\log \log x}}\right) *\right) \\
& =\sum_{k=3}^{x} \frac{1}{k}+O \int_{3}^{x} \frac{1}{u} e^{-c \sqrt{\log \log u}} d u-\log x+O\left(\log x e^{-c \sqrt{\log \log x}}\right) \\
& =\log x+O(1)+O \int_{\log 3}^{\log x} e^{-c \sqrt{\log y}} d y-\log x+O\left(\log x e^{-c \sqrt{\log \log x}}\right) \\
& =O\left(\log x e^{-c \sqrt{\log \log x}}\right) .
\end{aligned}
$$

Die Gleichung (10) verwandelt sich demnach in

$$
\begin{gathered}
\frac{1}{2} \log ^{2} x g(x)+\log x+O\left(\log x e^{-c \sqrt{\log \log x}}\right)=\log x+O(1) \\
\log ^{2} x g(x)=O\left(\log x e^{-c \sqrt{\log \log x}}\right) \\
g(x)=O\left(\frac{1}{\log x e^{e \sqrt{\log \log x}}}\right)
\end{gathered}
$$

Bei passender Wahl zweier positiver Constanten $e$ und $\gamma$ ist also für alle $\left.x \geqq e^{* * *}\right)$

$$
\log x e^{c \sqrt{\log \log x}}\left|\sum_{k=1}^{x} \frac{\mu(k)}{k}\right| \leqq \gamma .
$$

*) Für $k=1$ und $k=2$ wäre $\sqrt{\log \log k}$ imaginär; durch Weglassung der beiden ersten Summenglieder entsteht aber nur ein endlicher Fehler, der gegen $\log x e^{-c \sqrt{\log \log x}}$ vernachlässigt werden kann.

*) Für $x<e$ wäre die linke Seite imaginär. 


\section{$\S 4$.}

Daraus folgt, dass die von Herm von Mangoldt*) mit $\boldsymbol{M}(x)$ und von Herrn Mertens $\left.{ }^{* *}\right)$ mit $\sigma(x)$ bezeichnete Function $\sum_{k=1}^{x} \mu(k)$ höchstens von der Grössenordnung

ist; denn

$$
\frac{x}{\log x e^{\cos \sqrt{\log \log x}}}
$$

$$
\begin{gathered}
M(x)=\sum_{k=1}^{x} \mu(k)=\sum_{k=1}^{x} \frac{\mu(k)}{k} \cdot k=\sum_{k=1}^{x}(g(k)-g(k-1)) k \\
=\sum_{k=1}^{x} g(k)(k-(k+1))+g(x)([x]+1) \\
=-\sum_{k=1}^{x} g(k)+g(x)([x]+1) \\
=O \int_{e}^{x} \frac{d u}{\log u e^{c \sqrt{\log \log u}}}+O\left(\frac{x}{\log x e^{c \sqrt{\log \log x}}}\right)=O\left(\frac{x}{\log x e^{c \sqrt{\log \log x}}}\right) .
\end{gathered}
$$

Unter den unterhalb $x$ gelegenen quadratfreien Zahlen, deren Anzahl bekanntlich

$$
\begin{aligned}
q(x) & =\sum_{n=1}^{\sqrt{x}} \mu(n)\left[\frac{x}{n^{2}}\right]=\sum_{n=1}^{\sqrt{x}} \mu(n)\left(\frac{x}{n^{2}}+O(1)\right)=x \sum_{n=1}^{\sqrt{x}} \frac{\mu(n)}{n^{2}}+O(\sqrt{x}) \\
& =x \sum_{n=1}^{\infty} \frac{\mu(n)}{n^{2}}+x O \sum_{\sqrt{x}}^{\infty} \frac{1}{n^{2}}+O(\sqrt{x})=\frac{6}{\pi^{2}} x+O(\sqrt{x})
\end{aligned}
$$

beträgt, giebt es also nicht nur asymptotisch ebensoviele, die aus einer geraden als solche, die aus einer ungeraden Anzahl von Primfactoren zusammengesetzt sind ${ }^{* * *}$ ), sondern es lässt sich über die Differenz dieser beiden Anzahlen (deren jede asymptotisch gleich $\frac{3}{\pi^{2}} x$ ist) aussagen, dass sie höchstens von der Ordnung $\frac{x}{\log x e^{c \sqrt{\log \log x}}}$ unendlich wird.

*) 1. c., S. 850 .

* "Ueber eine zahlentheoretische Function", Sitzungsberichte der Wiener Akademie, Bd. 106, Abt. 2a, 1897, S. 761.

***) Dies folgt schon aus der Convergenz der Reihe $\sum_{k=1}^{\infty} \frac{\mu(k)}{k}$; s. v. Mangoldt, S. 849-851 und des Verf. „Neuer ete.", S. 15-16. 
Wenn $\pi(x)$ die Anzahl der Primzahlen $\leqq x$ und allgemeiner $\boldsymbol{\pi}_{\nu}(x)$ die Anzahl aller Zahlen $\leqq x$ bezeichnet, welche quadratfrei und aus $\nu$ Primfactoren zusammengesetzt sind, so ist, wie ich a. a. 0 . gezeigt habe*),

$$
\pi_{\nu}(x)=\frac{1}{(\nu-1) !} \frac{x(\log \log x)^{\nu-1}}{\log x}+O\left(\frac{x(\log \log x)^{\nu-2}}{\log x}\right) .
$$

Hier hat sich eine asymptotische Abschätzung von

$$
1-\pi_{1}(x)+\pi_{2}(x)-\cdots+(-1)^{v} \pi_{v}(x)+\cdots
$$

ergeben, da dieser Ausdruck mit $\sum_{k=1}^{x} \mu(k)$ übereinstimmt, während

$$
1+\pi_{1}(x)+\pi_{2}(x)+\cdots+\pi_{v}(x)+\cdots=q(x)=\frac{6}{\pi^{2}} x+o(\sqrt{x})
$$
ist.

Es bezeichne $\lambda(k)$ im Anschluss an Liouville $\%$ ) +1 oder - 1, je nachdem $k$ aus einer geraden oder ungeraden Anzahl von Primfactoren besteht, mehrfache Primfactoren mehrmals gerechnet. Dann ist bekanntlich

$$
\lambda(k)=\sum_{m} \mu\left(\frac{k}{m^{2}}\right)
$$

wo $m^{2}$ alle quadratischen Theiler von $k$ durchläuft; denn $\mu\left(\frac{k}{m^{2}}\right)$ ist für den grössten quadratischen Theiler $m^{2}$ von $k$ gleich $(-1)^{\omega}$, wo $\omega$ die Anzahl der verschiedenen Primfactoren von $k$ bezeichnet, und es ist stets

$$
\lambda\left(m^{2} n\right)=\lambda(n)
$$

für die anderen quadratischen Theiler $m^{2}$ von $k$ ist aber $\frac{k}{m^{2}}$ nicht quadratfrei, also

$$
\mu\left(\frac{k}{m^{2}}\right)=0
$$

Demnach ist

$$
L(x)=\sum_{k=1}^{x} \lambda(k)=\sum_{k=1}^{x} \sum_{m} \mu\left(\frac{k}{m^{2}}\right)=\sum_{m=1}^{\sqrt{x}} \sum_{k} \mu\left(\frac{k}{m^{2}}\right),
$$

wo $k$ alle Zahlen durchläuft, welche durch $m^{2}$ theilbar sind und unterhalb $x$ liegen, d. h., wie auch direct ersichtlich ist,

*) "Sur quelques problèmes relatifs à la distribution des nombres premiers", Bulletin de la société mathématique de France, t. 28, 1900, p. 37.

*) "Sur quelques fonctions numériques", Journal de mathématiques pures et * appliquées, $2^{\text {e }}$ série, t. 2,1857, p. 246. 


$$
\begin{aligned}
L(x) & =\sum_{m=1}^{\sqrt{x}} \sum_{n=1}^{\frac{x}{m^{2}}} \mu(n)=\sum_{m=1}^{\sqrt{x}} M\left(\frac{x}{m^{2}}\right)=\sum_{m=1}^{\log ^{2} x} M\left(\frac{x}{m^{2}}\right)+\sum_{\log ^{2} x}^{\sqrt{x}} M\left(\frac{x}{m^{2}}\right) \\
& =O \sum_{m=1}^{\log ^{2} x} \frac{x}{m^{2}(\log x-2 \log m) e^{c \sqrt{\log (\log x-2 \log m}}+O \sum_{\log ^{2} x}^{\sqrt{x}} \frac{x}{m^{2}}}+x \\
& =O \sum_{m=1}^{\log ^{2} x} \frac{x}{m^{2}(\log x-4 \log \log x) e^{c \sqrt{\log (\log x-4 \log \log x}}}+\infty \sum_{\log ^{2} x}^{\infty} \frac{1}{m^{2}} \\
& =O\left(\frac{x}{\log x e^{c \sqrt{\log \log x}}} \sum_{m=1}^{\infty} \frac{1}{m^{2}}\right)+O\left(\frac{x}{\log x}\right) \\
& =O\left(\frac{x}{\log x e^{c \sqrt{\log \log x}}}\right),
\end{aligned}
$$

so dass nicht nur, wie schon Herr von Mangoldt*) gefunden hat, es bis $x$ asymptotisch ebensoviele Zahlen giebt, die aus einer geraden, als solche, die aus einer ungeraden Anzahl von Primfactoren zusammengesetzt sind, sondern sogar die Differenz beider Anzahlen von angebbar geringerer Gxössenordnung unendlich wird. Bezeichnet $\sigma_{\nu}(x)$ die Anzahl aller Zahlen $\leqq x$, welche aus $\nu$ Primfactoren zusammengesetzt sind, mehrfache mehrmals gerechnet, so ist

$$
\left.\sigma_{\nu}(x)=\frac{1}{(\nu-1) !} \frac{x(\log \log x)^{\nu-1}}{\log x}+O\left(\frac{x(\log \log x)^{\nu-2}}{\log x}\right) * * *\right)
$$

$1-\sigma_{1}(x)+\sigma_{2}(x)-\cdots+(-1)^{\nu} \sigma_{\nu}(x)+\cdots=\sum_{k=1}^{x} \lambda(k)=O\left(\frac{x}{\log x e^{c \sqrt{\log \log x}}}\right)$, $1+\sigma_{1}(x)+\sigma_{2}(x)+\cdots+\sigma_{\nu}(x)+\cdots=[x]=x+O(1)$.

Mit Hilfe der erhaltenen Abschätzung für $g(x)$ lassen sich Ungleichungen für Ausdrücke der Form

$$
\sum_{k=1}^{x} \frac{\mu(k)}{k} F(k)=\sum_{k=1}^{x} g(k)(F(k)-F(k+1))+g(x) F([x]+1)
$$

aufstellen. Im Besonderen ergiebt sich die absolute Convergenz aller Reihen der Form

$$
\sum_{k=1}^{\infty} g(k) G(k)
$$

*) 1. c., S. 852 .

**) ,Sur quelques etc." p. 38. 
wo $G(k)$ von einer gewissen Stelle $a$ an positiv ist und monoton abnimmt, ferner der Bedingung genügt, dass das Integral

$$
\int_{a}^{\infty} \frac{G(u)}{\log u e^{c \sqrt{\log \log u}}} d u
$$

einen Sinn hat. Dies ist für

der Fall, da

$$
G(u)=\frac{1}{u}
$$

also für $a>e$

$$
e^{-c \sqrt{\log \log u}}=O\left(\frac{1}{(\log \log u)^{2}}\right)
$$

$$
\int_{a}^{\infty} \frac{d u}{u \log u e^{c \sqrt{\log \log u}}}=O \int_{a}^{\infty} \frac{d u}{u \log u(\log \log u)^{2}}=O\left(\frac{1}{\log \log a}\right)=O(1)
$$

ist. Also convergirt die Reihe

$$
\sum_{k=1}^{\infty} \frac{g(k)}{k}
$$

absolut. Es ist jedoch zu bemerken, dass die bedingte Convergenz dieser Reihe schon aus (13) durch folgendes Verfahren hergeleitet werden kann. Es ist nach (13)

$$
\begin{aligned}
1+\{1\} & =\log x \sum_{k=1}^{x} \frac{\mu(k)}{k}-\sum_{k=1}^{x} \frac{\mu(k) \log k}{k}=\sum_{k=1}^{x} \frac{\mu(k)}{k} \log \frac{x}{k} \\
& =\sum_{k=1}^{x} \log \frac{x}{k}(g(k)-g(k-1)) \\
& =\sum_{k=1}^{x} g(k)\left(\log \frac{x}{k}-\log \frac{x}{k+1}\right)+g(x) \log \frac{x}{[x]+1} \\
& =\sum_{k=1}^{x} g(k) \log \left(1+\frac{1}{k}\right)+\{1\} \\
& =\sum_{k=1}^{x} g(k)\left(\frac{1}{k}-\frac{\vartheta_{k}}{2 k^{2}}\right)+\{1\} \quad\left(0 \leqq \vartheta_{k} \leqq 1\right) .
\end{aligned}
$$

Da $\left|g(k) \vartheta_{k}\right| \leqq 1$ ist, convergirt die Reihe $\sum_{k=1}^{\infty} \frac{g(k) \vartheta_{k}}{2 k^{2}}\left(\vartheta_{k}\right.$ ist für jedes $k$ eine ganz bestimmte Zahl zwischen 0 und 1 ); also convergirt, wie behauptet, die Reihe $\sum_{k=1}^{\infty} \frac{g(k)}{k}$. 
Analoge Sätze gelten über die mit Liouville'schen Coefficienten behafteten Reihen. Ich hebe besonders hervor, dass sich aus

mit Hilfe der Identität

$$
g(x)=\sum_{k=1}^{x} \frac{\mu(k)}{k}=O\left(\frac{1}{\log x e^{c \sqrt{\log \log x}}}\right)
$$

leicht ergiebt, dass

$$
G(x)=\sum_{m=1}^{\sqrt{x}} \frac{1}{m^{2}} g\left(\frac{x}{m^{2}}\right)
$$

$$
G(x)=\sum_{k=1}^{x} \frac{\lambda(k)}{k}=O\left(\frac{1}{\log x e^{c \sqrt{\log \log x}}}\right) *
$$

ist. Daraus folgt weiter, dass

$$
\sum_{k=1}^{x} \frac{\lambda(k) \log k}{k}
$$

sich einer Grenze nähert, genaver, dass das Restglied von der Ordnung $e^{-a \sqrt{\log \overline{\log x}}}$ ist. Der Werth dieser Grenze stimmt mit

überein.

$$
\begin{aligned}
\lim _{s=1} \sum_{k=1}^{\infty} \frac{\lambda(k) \log k}{k^{s}} & \left.=-\lim _{s=1} \frac{d}{d s} \sum_{k=1}^{\infty} \frac{\lambda(k)}{k^{s}}=-\lim _{s=1} \frac{d}{d s} \frac{\xi(2 s)}{\xi(s)}\right) \\
& =-\lim _{s=1}\left(-\frac{\xi(2 s) \zeta(s)}{\xi^{2}(s)}+\frac{2 \zeta(2 s)}{\zeta(s)}\right)=-\xi(2)=-\frac{\pi^{2}}{6}
\end{aligned}
$$

Noch zwei asymptotische Folgerungen mögen hier Platz finden, weil sie sich auf Summen beziehen, bei denen der absolute Betrag des Summanden sich nicht monoton ändert, bei deren Behandlung also das gewöhnliche, von Abel herrührende Hilfsmittel der partiellen Summation versagt.

Bezeichnet $\varrho(x)$ den in $x$ enthaltenen echten Bruch, also die Function $x-[x]$, so ist erstens zufolge einer schon von Meissel***) mit ungenügendem Beweise ausgesprochenen und von Herm Lipschitz $\dagger$ ) bewiesenen Identität

*) Die Gleichung $\sum_{k=1}^{\infty} \frac{\lambda(k)}{k}=1-\frac{1}{2}-\frac{1}{3}+\frac{1}{4}-\frac{1}{5}+\frac{1}{6}-\cdots=0$ ist auch schon von Euler auf heuristischem Wege hergeleitet worden, 1. c, S. 229.

**) S. Cantor, „zur Theorie der zahlentheoretischen Functionen", Mathematische Annalen Bd. 16, 1880, S. 586, aber auch schon Euler, 1. c., S. 228.

**) „Observationes quaedam in theoria numeroram", Journal für die reine and angewandte Mathematik, Bd. 48, 1854, S. 303.

†) "Sur des séries relatives à la théorie des nombres", Comptes rendus des séances de l'académie des sciences, Paris, t. 89, 1879, p. 949. 


$$
\begin{gathered}
1=\sum_{k=1}^{x} \mu(k)\left[\frac{x}{k}\right]=\sum_{k=1}^{x} \mu(k)\left(\frac{x}{k}-\varrho\left(\frac{x}{k}\right)\right)=x g(x)-\sum_{k=1}^{x} \mu(k) \varrho\left(\frac{x}{k}\right) \\
\sum_{k=1}^{x} \mu(k) \varrho\left(\frac{x}{k}\right)=O\left(\frac{x}{\log x e^{c \sqrt{\log \log x}}}\right) .
\end{gathered}
$$

Zweitens besteht, wenn $\psi(x)$ die von Tschebyschef*) eingeführte Bedeutung

$$
\psi(x)=\vartheta(x)+\vartheta(\sqrt{x})+\vartheta(\sqrt[3]{x})+\cdots=\sum_{p \leq x} \log p\left[\frac{\log x}{\log p}\right]=\sum_{k=1}^{x} \mu(k) \log \left(\left[\frac{x}{k}\right] !\right)
$$

hat, die Identität**)

$$
\sum_{k=1}^{x} \mu(k)\left[\frac{x}{k}\right] \log k=-\psi(x)
$$

Auf die Analogie der beiden damals noch unbewiesenen Sätze

und

$$
\lim _{x=\infty} \frac{-\psi(x)}{x}=\lim _{x=\infty} \frac{1}{x} \sum_{k=1}^{x} \mu(k)\left[\frac{x}{k}\right] \dot{\log } k=-1
$$

$$
\lim _{x=\infty} f(x)=\lim _{x=\infty} \sum_{k=1}^{x} \mu(k) \frac{1}{k} \log k=\lim _{x=\infty} \frac{1}{x} \sum_{k=1}^{x} \mu(k) \frac{x}{k} \log k=-1
$$

hat schon Herr Gram**) hingewiesen. Aus der von Herrn de la ValléePoussin bewiesenen Relation

$$
\vartheta(x)=x+O\left(x e^{-b \sqrt{\log x}}\right)
$$

folgt in Verbindung mit der schon von Tschebyschef $\dagger$ ) gemachten Bemerkung, dass sich $\psi(x)$ und $\vartheta(x)$ nur um eine Grösse $O(\sqrt{x})$ unterscheiden,

Demnach ist

$$
\psi(x)=x+O\left(x e^{-b \sqrt{\log x}}\right)
$$

$$
\begin{gathered}
x+O\left(x e^{-b \sqrt{\log x}}\right)=-\sum_{k=1}^{x} \mu(k)\left[\frac{x}{k}\right] \log k=-\sum_{k=1}^{x} \mu(k) \log k\left(\frac{x}{k}-\varrho\left(\frac{x}{k}\right)\right) \\
=-x f(x)+\sum_{k=1}^{x} \mu(k) \log k \varrho\left(\frac{x}{k}\right)=x+O\left(x e^{-c \sqrt{\log \log x}}\right)+\sum_{k=1}^{x} \mu(k) \log k \varrho\left(\frac{x}{k}\right), \\
(22) \quad \sum_{k=1}^{x} \mu(k) \log k \varrho\left(\frac{x}{k}\right)=O\left(\frac{x}{e^{c \sqrt{\log \log x}}}\right) .
\end{gathered}
$$

*) "Mémoire sur les nombres premiers", Journal de mathématiques pures et appliquées, $1^{\text {ère }}$ série, t. 17,1852 , p. 371.

*) S. Gram, 1. c., S. 238.

**) 1. c., S. 238 und 299.

†) 1.c., S. 378. 
Die Summen (21) und (22) sind also von weit geringeren Grössenordnungen als man durch das primitivste Abschätzungsverfahren

$$
\left|\mu(k) \rho\left(\frac{x}{k}\right)\right| \leqq 1
$$

erhält (nämlich $O(x)$ bezw. $O(x \log x)$ ); dagegen weiss man von der Summe

$$
\sum_{k=1}^{x} \mu(k) \frac{1}{k} \varrho\left(\frac{x}{k}\right)
$$

nichts ausser der selbstverständlichen Thatsache, dass sie höchstens von der Ordnung $\log x$ ist. Diese Summe spielt bei der Bestimmung des mittleren Werthes der Function $\varphi(n)$ eine Rolle. Herr Mertens*) ist bis zu der Gleichung

$$
\sum_{n=1}^{x} \varphi(n)=\frac{3}{\pi^{2}} x^{2}-x \sum_{k=1}^{x} \mu(k) \frac{1}{k} \rho\left(\frac{x}{k}\right)+O(x)
$$

gelangt, aus der er die Folgerung

$$
\sum_{n=1}^{x} \varphi(n)=\frac{3}{\pi^{2}} x^{2}+O(x \log x)
$$

gezogen hat; eine genauere Abschätzung der Summe (23) würde einen Fortschritt in dieser Frage mit sich führen.

Juni 1900.

*) „Ueber einige asymptotische Gesetze in der Zahlentheorie“, Journal für die reine und angewandte Mathematik, Bd. 77, 1874, S. 290. 